ARTICLE

\title{
Regulating strain in perovskite thin films through charge-transport layers
}

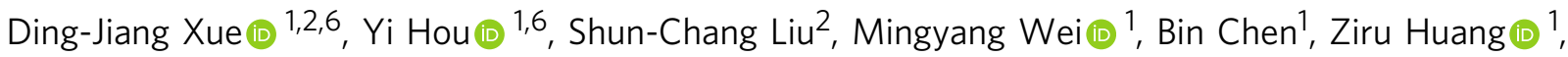 \\ Zongbao Li ${ }^{2,3}$, Bin Sun (D) ${ }^{1}$, Andrew H. Proppe (10) ${ }^{1,4}$, Yitong Dong ${ }^{1}$, Makhsud I. Saidaminov ${ }^{1}$, Shana O. Kelley (1D) 4,5, \\ Jin-Song Hu (i) ${ }^{2} \&$ Edward H. Sargent (i) ${ }^{1 凶}$
}

Thermally-induced tensile strain that remains in perovskite films following annealing results in increased ion migration and is a known factor in the instability of these materials. Previously-reported strain regulation methods for perovskite solar cells (PSCs) have utilized substrates with high thermal expansion coefficients that limits the processing temperature of perovskites and compromises power conversion efficiency. Here we compensate residual tensile strain by introducing an external compressive strain from the hole-transport layer. By using a hole-transport layer with high thermal expansion coefficient, we compensate the tensile strain in PSCs by elevating the processing temperature of hole-transport layer. We find that compressive strain increases the activation energy for ion migration, improving the stability of perovskite films. We achieve an efficiency of $16.4 \%$ for compressively-strained PSCs; and these retain $96 \%$ of their initial efficiencies after heating at $85^{\circ} \mathrm{C}$ for 1000 hoursthe most stable wide-bandgap perovskites (above $1.75 \mathrm{eV}$ ) reported so far.

\footnotetext{
${ }^{1}$ Department of Electrical and Computer Engineering, University of Toronto, Toronto, ON M5S 1A4, Canada. ${ }^{2}$ Beijing National Laboratory for Molecular Sciences (BNLMS), CAS Key Laboratory of Molecular Nanostructure and Nanotechnology, Institute of Chemistry, Chinese Academy of Sciences, 100190 Beijing, China. ${ }^{3}$ National Engineering Research Center for Advanced Polymer Processing Technology, Zhengzhou University, 450002 Zhengzhou, China. ${ }^{4}$ Department of Chemistry, University of Toronto, Toronto, ON M5S 3G4, Canada. ${ }^{5}$ Leslie Dan Faculty of Pharmacy, Department of Pharmaceutical Sciences, University of Toronto, Toronto, ON M5S 3M2, Canada. ${ }^{6}$ These authors contributed equally: Ding-Jiang Xue, Yi Hou. ${ }^{\bowtie}$ email: ted.sargent@utoronto.ca
} 
T he power conversion efficiencies (PCEs) of perovskite solar cells (PSCs) have increased from $3.8 \%^{1}$ to a certified $25.2 \%{ }^{2}$, approaching those of crystalline silicon solar cells. Continued progress in stability remains a topic of importance along the path to industrial application ${ }^{3-5}$. Encapsulating PSCs to protect against oxygen and moisture is a straightforward method to stabilize these devices ${ }^{6-8}$; however, some important sources of instability are not overcome using extrinsic stabilization approaches such as encapsulation?.

Residual tensile strain in perovskite films is a source of instability, and stems from the thermal expansion mismatch between perovskites and substrates during the annealing steps required in the formation of perovskites ${ }^{10-13}$. Specifically, thermally-induced tensile stresses in perovskite films can reach $50 \mathrm{MPa}$, sufficient to induce the deformation of copper ${ }^{12}$. These tensile stresses weaken bonds, decreasing the formation energy of defects and lowering the activation energy for ion migration ${ }^{9,11}$. These mechanisms are obstacles to stability at elevated temperatures, such as those required in accelerated lifetime testing.

The correlation between stress $(\sigma)$ and thermal expansion mismatch is quantified as follows:

$$
\sigma_{\Delta T}=\frac{E_{\mathrm{p}}}{1-v_{\mathrm{p}}}\left(\alpha_{\mathrm{s}}-\alpha_{\mathrm{p}}\right) \Delta T
$$

where $E_{\mathrm{p}}$ is the modulus of the perovskite, $v_{\mathrm{p}}$ is Poisson's ratio in the perovskite, $\alpha_{\mathrm{s}}$ and $\alpha_{\mathrm{p}}$ are the thermal expansion coefficients of the substrate and the perovskite, respectively, and $\Delta T$ is the temperature gradient during cooling from the annealing temperature of the perovskite film to room temperature ${ }^{12}$. Several strategies have been reported to reduce this detrimental tensile strain in perovskite films based on the above equation ${ }^{11,12}$. They can be divided into two categories: (i) lowering the formation temperature of perovskite films to reduce $\Delta T^{12}$; (ii) using plastic substrates with thermal expansion coefficients that are similar to that of the perovskite to decrease $\Delta \alpha^{11}$. Although these strategies have been shown to diminish the residual tensile stress in perovskite films, they also lower device efficiency. Roomtemperature-prepared $\mathrm{CH}_{3} \mathrm{NH}_{3} \mathrm{PbI}_{3}$-based solar cells exhibit a PCE of $17.1 \%{ }^{14}$, a result of the lower quality of perovskite films fabricated by low-temperature processing. It is imperative to control the residual stress to move further in the direction of high efficiency and increased stability of PSCs.

Here we report a strain-compensation strategy that reduces the tensile strain in perovskite films with the aid of hole-transport layer (HTL). We use an HTL possessing numerous carbonyl anchoring groups that exhibit strong interactions with the perovskite surfaces, and thus we build a strong HTL: perovskite interface that transfers strain from the HTL to the perovskite active layer; we then balance the tensile/compressive strain transition by tuning the processing temperature and strain of the HTL. The resultant compressively-strained photovoltaic devices retain $95 \%$ of their initial PCEs of $16.4 \%$ after maximum power point tracking for $60 \mathrm{~h}$, and $96 \%$ after heating at $85^{\circ} \mathrm{C}$ for $1000 \mathrm{~h}$, showing the most stable wide-bandgap perovskite (above $1.75 \mathrm{eV}$ ) cells reported so far. The improved stability of compressivelystrained material stacks is explained through the higher activation energy (from 0.547 to $0.794 \mathrm{eV}$ ) for ion migration compared to that of tensile-strained films.

\section{Results}

Residual strain in perovskite films. Based on Eq. (1), the difference in thermal expansion coefficients $(\alpha)$ between the perovskite and the contacting layers provides one source of stress; and the requirement of high annealing temperatures to form crystalline perovskites also contributes due to a large $\Delta T$.
Regarding the thermal expansion mismatch, PSCs typically consist of a stack of multilayers including a substrate coated with a transparent conducting oxide electrode (TCO) layer, followed by the electron-transport layer (ETL), the perovskite layer, the holetransport layer (HTL), and another electrode layer. The thermal expansion coefficient varies greatly with each functional layer (Fig. 1a). The widely used ITO-coated glass and metal oxide charge-transport layers possess low values of $\alpha$ in the range of 0.37 to $1 \times 10^{-5} \mathrm{~K}^{-115}$. In contrast, perovskites have much higher a values ranging from 3.3 to $8.4 \times 10^{-5} \mathrm{~K}^{-116-18}$, approximately an order of magnitude higher than that of substrates or ETLs. Such a large thermal expansion difference is the main reason for the formation of tensile strain as perovskite films cool to room temperature ${ }^{11,12}$.

As shown in Fig. 1b, the perovskite films used for highefficiency perovskite solar cells typically require annealing at temperatures over $100^{\circ} \mathrm{C}$ for improved crystallinity and minimization of defects ${ }^{19-23}$. Notably, when comparing with hybrid organic-inorganic perovskites, all-inorganic perovskites require even higher temperatures to stabilize the black cubic perovskite phase. In particular, $\mathrm{CsPbI}_{3}$ requires annealing temperatures varying from 180 to $330^{\circ} \mathrm{C}^{19,24}$. Therefore, inorganic perovskite films processed at high temperatures suffer from an even larger tensile strain.

When a perovskite film is deposited on a layer with lower $\alpha$, the contact formed between the two layers during the hightemperature annealing process constrains the perovskite from contracting when it cools back to room temperature, introducing tensile strain along the in-plane direction (Fig. 1c). Conversely, when using a layer with higher $\alpha$, the perovskite film contracts more, resulting in compressive strain. Therefore, tensile or compressive strain in perovskite films can be adjusted by using adjacent device layers with lower or higher $\alpha$ compared with that of perovskite.

We propose a strain-management approach to reduce residual tensile strain in perovskite films-since tensile strain is more often produced in these layer stacks than compressive strain. When using a substrate with lower $\alpha$, we are able to compensate the tensile strain through an external compressive strain that is induced by the top contacting HTL with higher $\alpha$, leading to a non-strained or even compressively-strained perovskite film.

Strain-compensated perovskite films. To evaluate this straincompensation strategy experimentally, we focused on allinorganic cesium lead halide perovskites primarily due to their superior thermal stability compared to their organic-inorganic hybrid counterparts ${ }^{19,20}$. This high thermal stability enables allinorganic perovskites to withstand higher annealing temperatures (Fig. 1b), while also inducing a large amount of stress considering the soft lattice of perovskites that can endure large strain (Fig. 1d and Supplementary Fig. 1), thereby facilitating the investigation of strain control. Specifically, we chose $\mathrm{CsPbI}_{2} \mathrm{Br}$ as a suitable candidate to study the strain engineering in perovskite films since it is a more stable photoactive perovskite cubic structure, and it provides a wide bandgap of $1.92 \mathrm{eV}^{25-28}$, potentially serving as top cells in tandem devices with silicon or narrow-bandgap perovskite solar cells.

We took the view that functional layers suitable for our straincompensation strategy would need to meet the following three criteria: (i) the top functional layer should have a higher thermal expansion coefficient compared with the perovskite, offering the possibility of compressive strain; (ii) the functional layer should have a strong interaction with the perovskite in order to anchor to the lattice and achieve strain offset; (iii) the top interface layer should be coated at high temperatures, inducing a compressive 


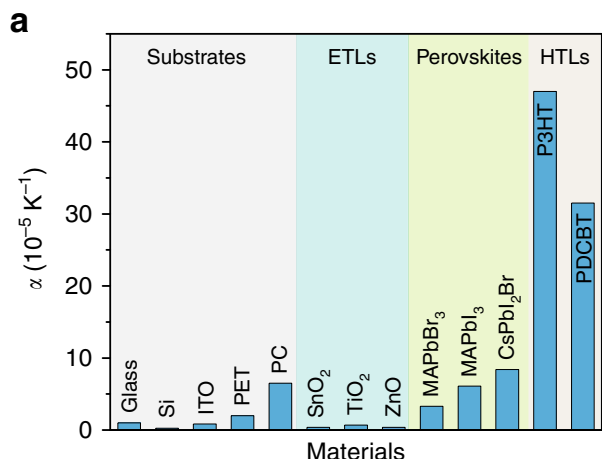

C

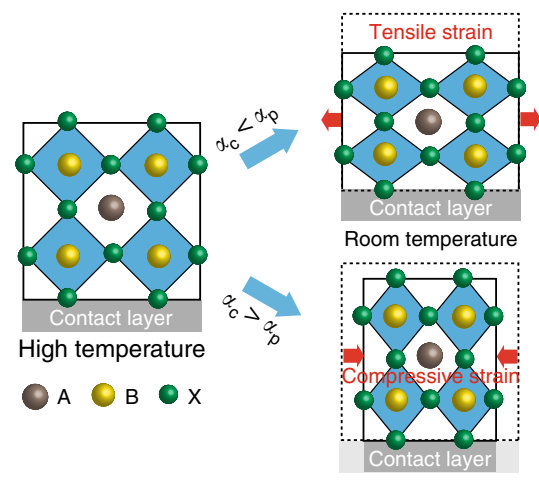

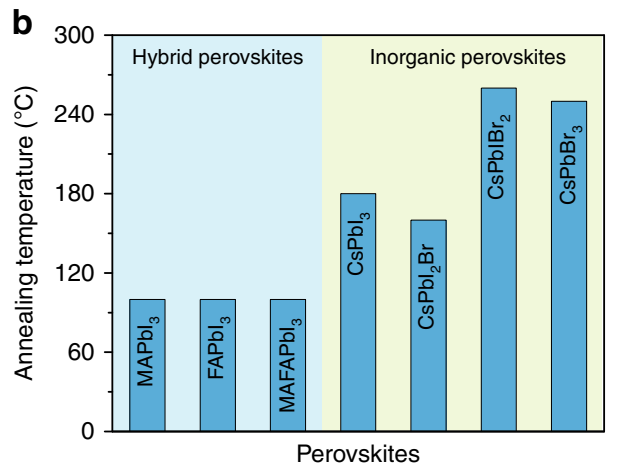

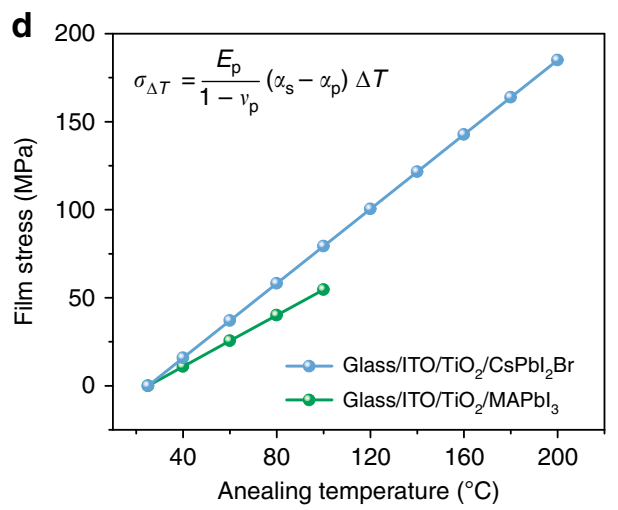

Fig. 1 Analysis of thermally-induced strain in perovskite films. a Thermal expansion coefficients of widely-used functional layers in PSCs including substrates, ETLs, perovskites, and HTLs. b Annealing temperatures of different hybrid and inorganic perovskite films during formation. c Schematic showing the formation of tensile and compressive strains. $\mathbf{d}$ The calculated annealing temperature-dependent stress from thermal expansion mismatch between the substrate and perovskites including $\mathrm{MAPbl}_{3}$ and $\mathrm{CsPbl}_{2} \mathrm{Br}$.

strain when cooling back to room temperature, according to the above equation relating stress and thermal expansion mismatch.

In this regard, we believed that poly[5,5-bis(2-butyloctyl)-(2,2bithiophene)-4,4'-dicarboxylate-alt-5, $5^{\prime}-2,2^{\prime}$-bithiophene] (PDCBT) with rich carbonyl groups, a well-known polymer that can serve as a highly efficient HTL for PSCs ${ }^{29,30}$, and with a similar chemical structure to poly(3-hexylthiophene-2,5-diyl) (P3HT) (Supplementary Fig. 2), fulfilled the above criteria and would offer a compressive strain to offset the residual tensile strain in perovskite films. We first performed temperaturedependent X-ray diffraction (XRD) to measure the thermal expansion coefficient of PDCBT. The $\alpha$ of PDCBT was calculated to be $31.5 \times 10^{-5} \mathrm{~K}^{-1}$ through the shift of XRD peaks (Fig. 2a and Supplementary Fig. 3 ). This value was close to that of $\mathrm{P}_{3} \mathrm{HT}^{31-33}$ and more than an order of magnitude higher than that of $\mathrm{CsPbI}_{2} \mathrm{Br}$ (Fig. 2a and Supplementary Fig. 4), providing a large thermal expansion mismatch with the perovskite.

We then carried out X-ray photoelectron spectroscopy (XPS) to evaluate the interaction between PDCBT and the perovskite. Due to the shallow probing depth of XPS (about $10 \mathrm{~nm}$ ), a thin layer of PDCBT (less than $8 \mathrm{~nm}$ ) was coated on the top of perovskite film for the measurement. We observed that the $\mathrm{Pb} 4 f$ peaks shifted towards lower binding energy after PDCBT coating (Fig. 2b). In addition to the $\mathrm{O} 1 s$ peak from PDCBT at $531.5 \mathrm{eV}$ (as observed in pure PDCBT films), there is another O $1 s$ peak from perovskite/PDCBT film, which shifted towards a higher binding energy at $532.9 \mathrm{eV}$ (Fig. 2c). The S $2 p$ peaks remained constant when compared with pure PDCBT (Fig. 2d). Therefore, the XPS results demonstrated the strong interaction between the perovskite and PDCBT through the formation of $\mathrm{Pb}-\mathrm{O}$ bonds, consistent with our electrostatic potential analysis calculated using density functional theory (DFT), where the electronwithdrawing atom was $\mathrm{O}$, providing favorable condition for the passivation of under-coordinated $\mathrm{Pb}$ in the perovskite ${ }^{34,35}$ (Supplementary Fig. 5). Moreover, the introduction of PDCBT resulted in an evident blue-shifted photoluminescence (PL) emission peak for the perovskite film compared to that of P3HT and Spiro-OMeTAD (Fig. 2e), further indicating the efficient passivation effect of PDCBT while confirming the strong binding between perovskite and $\mathrm{PDCBT}^{36}$, in good agreement with XPS results.

We carried out further experiments varying the coating temperature of PDCBT layer, a key processing parameter for our strain-compensation strategy. Cl-capped $\mathrm{TiO}_{2}\left(\mathrm{TiO}_{2}-\mathrm{Cl}\right)$ nanocrystal films were used as an ETL according to previous work ${ }^{37,38}$; $\mathrm{CsPbI}_{2} \mathrm{Br}$ films were formed by annealing at $160^{\circ} \mathrm{C}$ for 10 min (Supplementary Fig. 6), resulting in a tensile stress of about $150 \mathrm{MPa}$ (Supplementary Fig. 1). The possible strain induced by local lattice mismatches can be excluded from the observed compositional homogeneity in $\mathrm{CsPbI}_{2} \mathrm{Br}$ films along the lateral (Supplementary Fig. 7) and vertical directions (Supplementary Fig. 8), as well as the little variation of lattice constant from the surface to the bottom (Supplementary Fig. 9). The residual strain in the perovskite films was insensitive to postannealing treatment due to the strong adhesion between perovskite and substrate once the perovskite is formed, according to a previous report ${ }^{11}$. Therefore, according to our straincompensation strategy, when coating PDCBT at high temperatures, the resulting compressive strain can offset the residual tensile strain. Figure $2 \mathrm{f}$ shows a linear correlation between stress and coating temperature of the PDCBT. This is consistent with a reduction of tensile stress in the perovskite film when increasing the HTL coating temperature.

To verify this hypothesis, we used out-of-plane XRD to probe the total strain in perovskite films after depositing the HTL at different temperatures, evaluated through the XRD peak shift. 


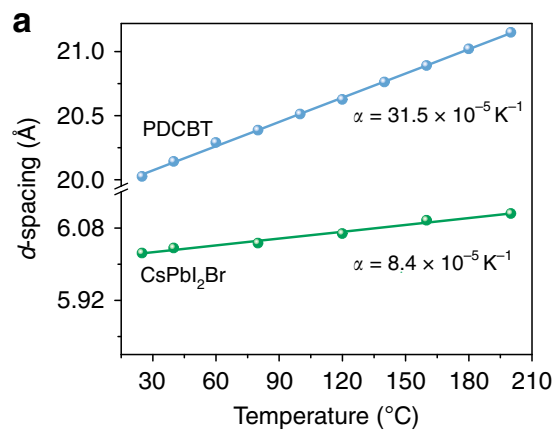

b

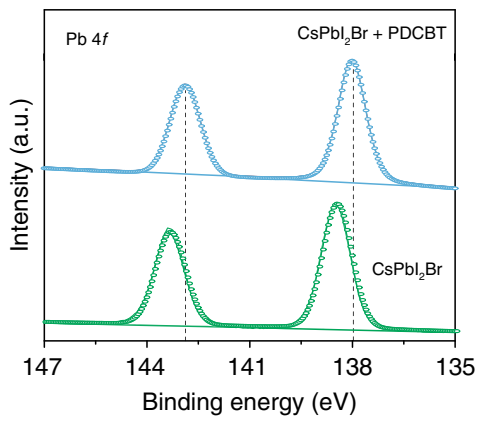

e

d

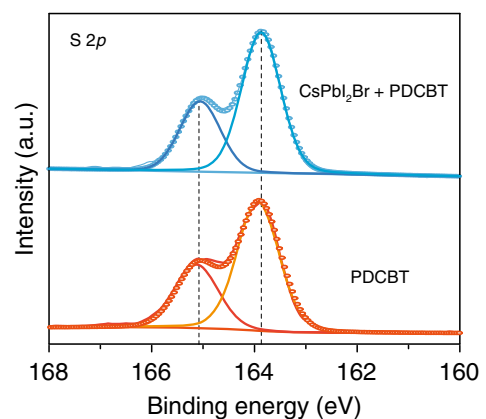

$\mathbf{g}$

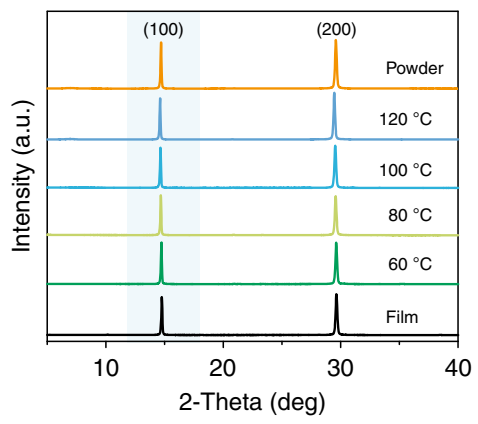

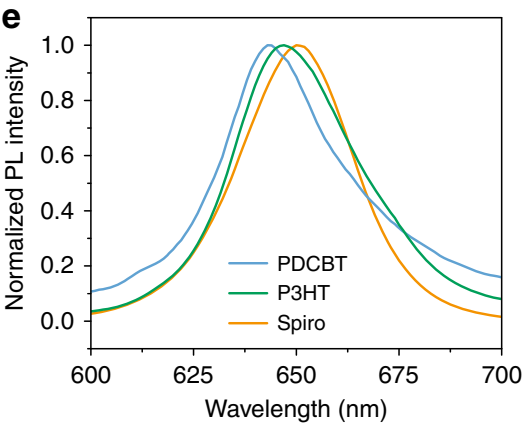

h

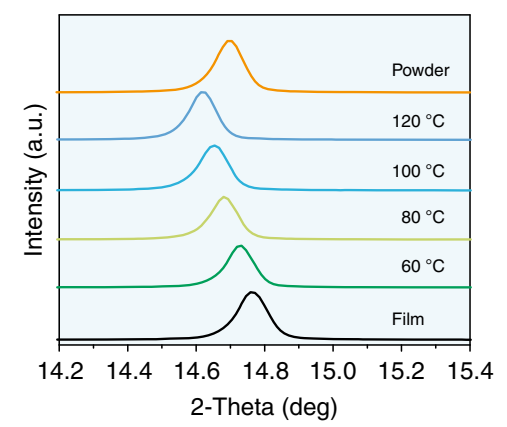

C
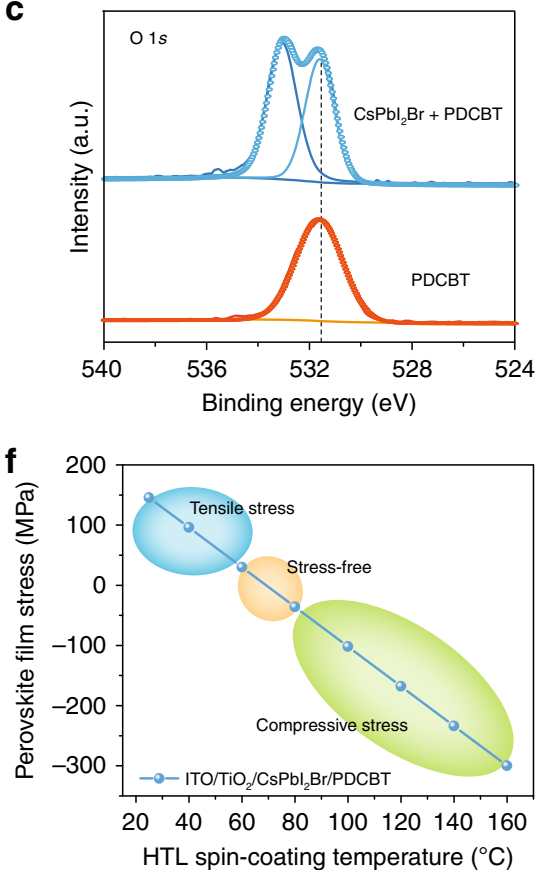

i

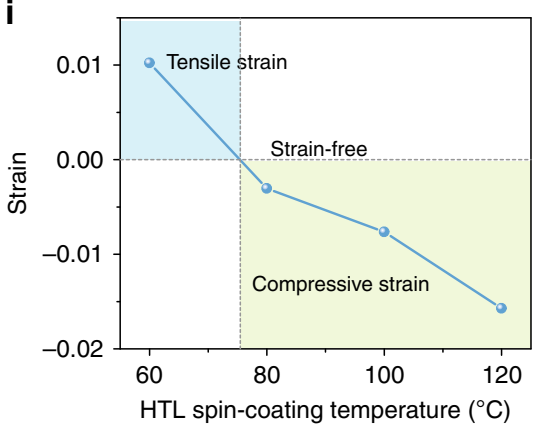

Fig. 2 Characterization of strain compensated perovskite thin films. a Temperature-dependent (100) d-spacing of PDCBT and perovskite films. XPS spectra of (b) Pb $4 f$, (c) O 1s and, (d) S $2 p$ in the perovskite, perovskite/PDCBT, and PDCBT films. e Normalized PL spectra of perovskite films with PDCBT, $\mathrm{P} 3 \mathrm{HT}$ and Spiro-OMeTAD from the ITO side. $\mathbf{f}$ The calculated net average stress in perovskites within structures consisting of ITO/TiO $/ 2$ perovskite/ PDCBT as a function of PDCBT spin-coating temperature. $\mathbf{g}$ XRD patterns of perovskite film, powder and perovskite/PDCBT films fabricated at different PDCBT spin-coating temperatures. h Magnified (100) diffraction peaks in the region indicated by the blue. i Measured strain in perovskite films coated with PDCBT HTLs at different spin-coating temperatures.

The XRD peaks of as-fabricated perovskite film shifted to higher diffraction angles compared to the strain-free peaks of scraped perovskite powder (Fig. $2 \mathrm{~g}$ ). Such a shift to higher angles can be attributed to the smaller plane spacing in the direction perpendicular to the substrate, thereby indicating the tensile strain in the horizontal direction of the film based on the positive Poisson's ratio in perovskites, in good agreement with previous reports ${ }^{9,11,12}$. Moreover, a gradual shift to lower diffraction angle is observed, as expected, and is shown in the magnified view of (100) peaks when increasing the HTL coating temperature (Fig. 2h and Supplementary Fig. 10). Note that the strain was nearly eliminated when the HTL coating temperature was approximately $80^{\circ} \mathrm{C}$. As the temperature is increased to $120^{\circ} \mathrm{C}$, the film becomes compressively strained (Fig. 2i). Furthermore, the comparison of XRD peaks between the perovskite/PDCBT film and the same perovskite film after the PDCBT is washed away indicates that the compressive strain stems from the PDCBT layer coated at high temperature (Supplementary Fig. 11). The depth-dependent grazing incidence XRD (GIXRD) results indicate the homogeneous distribution of compressive strain in this perovskite film (Supplementary Fig. 12). Consequently, these results demonstrate that the residual tensile strain in the perovskite film can be compensated by depositing the HTL atop the perovskite at high temperatures to introduce compressive strain, resulting in a strain-free or even compressively-strained perovskite film.

Stability of perovskite films under different strains. Such tunable strain in perovskite films, by controlling tension and compression, allows us to better investigate the correlation between film stress and the resulting stability. Despite the fact that perovskite vacancies are shallow electronic traps that do not act as recombination centers, they have been recently shown to strongly contribute to perovskite decomposition ${ }^{37}$. Vacancy-assisted migration of halide ions results in halide segregation in mixedhalide perovskites, and subsequently phase segregation ${ }^{9}$.

We first calculated the formation energies of halide vacancy under different strain conditions using DFT, which directly reflected the density of halide ion vacancies in perovskite. We found that the tensile strain decreased the formation energy of halide vacancies, while compressive strain increased their 
a

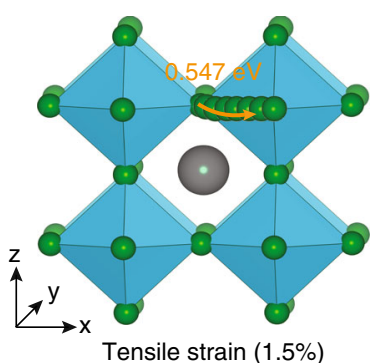

d

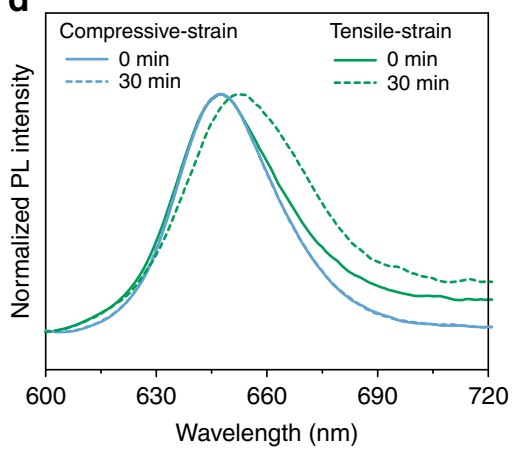

b

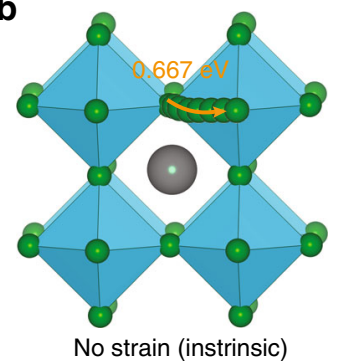

e

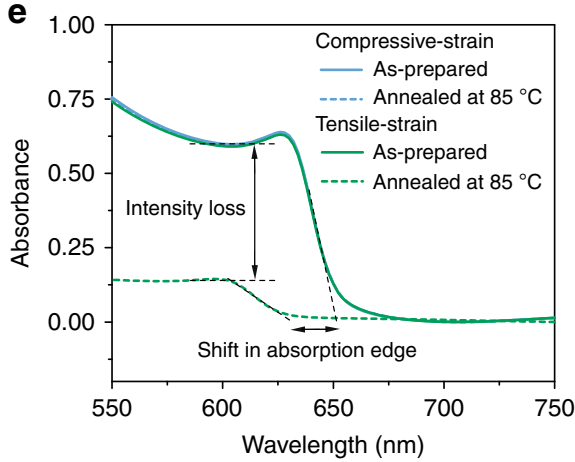

C

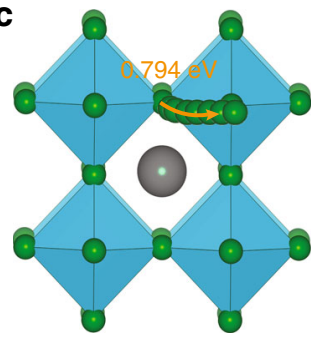

Compressive strain $(-1.5 \%)$

f

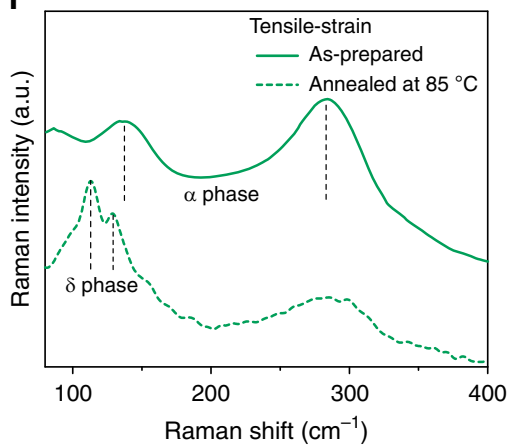

Fig. 3 Stability of perovskite films under different strains. Calculated activation energies for halide ion migration in perovskites under biaxial (a) 1.5\% tensile strain, (b) no strain and (c) $-1.5 \%$ compressive strain. $\mathbf{d}$ Normalized PL spectra of compressive-strain and tensile-strain perovskite films after illuminating for 0 and 30 min. e Absorption spectra of compressive-strain and tensile-strain perovskite films before and after annealing at $85^{\circ} \mathrm{C}$ for $60 \mathrm{~h}$. f Raman spectra of tensile-strain perovskite film before and after annealing at $85^{\circ} \mathrm{C}$ for $60 \mathrm{~h}$.

formation energy compared with intrinsic (strain-free) perovskites (Supplementary Fig. 13). Once halide vacancies are formed, we further calculated the relative activation energies for the vacancy-assisted migration of halide ions $\left(E_{\mathrm{a}}\right)$ in perovskites under biaxial tensile and compressive strains (Supplementary Fig. 13 and 14). The activation energy for halide ion migration in strain-free perovskite was $0.667 \mathrm{eV}$ (Fig. 3b), agreeing well with previous reports ${ }^{39,40}$; a lower activation energy of $0.547 \mathrm{eV}$ was found under biaxial tensile strain of $1.5 \%$ (Fig. 3a), whereas a higher energy of $0.794 \mathrm{eV}$ was found for biaxial compressive strain of $-1.5 \%$ (Fig. 3c). The above results indicate that compressive strain not only suppresses the formation of halide vacancies, but also decelerates ion migration in perovskites, improving their intrinsic stability.

To investigate the effect of strain on the stability of perovskite films from experiment, we studied the impact of strain in perovskite films on their rate of degradation. Mixed-halide $\mathrm{CsPbI}_{2} \mathrm{Br}$ perovskite films are known to undergo phase segregation into separated Br-rich and I-rich phases when exposed to light and heat, a consequence of increased ion migration activated under these conditions (Supplementary Fig. 15). This facilitated the measurement of halide ion migration ${ }^{21}$, which can be readily investigated through optical characterizations of Br-rich and I-rich phases.

We first investigated the photostability of perovskite films under compressive and tensile strains. According to Fig. 2e, the perovskite films coated with PDCBT at $120^{\circ} \mathrm{C}$ and $60^{\circ} \mathrm{C}$ were labeled as compressive-strain and tensile-strain perovskite films, respectively. After $30 \mathrm{~min}$ of continuous illumination, the compressive-strain film showed no change of PL peak position, whereas the tensile-strain film exhibited a redshift in the peak position (Fig. 3d), a signature of phase segregation. This improved photostability indicates that the compressive-strain film exhibits suppressed halide ion migration, in good agreement with our calculated results.

We then explored the thermal stability of compressive-strain and tensile-strain perovskite films. For the tensile-strain films, there are clear changes in the absorbance amplitude and a shift of absorption edge after annealing at $85^{\circ} \mathrm{C}$ in air for $60 \mathrm{~h}$ (Fig. 3e). This blueshift can be attributed to formation of Br-rich phase, while the loss absorption amplitude was due to the phase transition of I-rich phase from black a phase to transparent $\delta$ phase, indicative of severe halide ion migration at high temperatures ${ }^{21}$. Raman spectroscopy further confirmed the coexistence of a phase and $\delta$ phase perovskites ${ }^{22}$ (Fig. 3f). For compressive-strain film, the absorption spectrum remained unchanged after the same annealing process, exhibiting diminished ion migration under compressive strain. These findings agreed with our DFT results that compressive strain can suppress ion migration owing to high activation energy, improving the intrinsic stability of perovskites, similar to previous reports $9,11,12$.

Stability improvement in compressive-strain PSCs. To further investigate the effect of strain on device performance, three types of PSCs under different strains were fabricated with processing temperatures of the PDCBT HTL varied from $60^{\circ} \mathrm{C}$ (tensile strain), $90^{\circ} \mathrm{C}$ (strain free), and $120^{\circ} \mathrm{C}$ (compressive strain). We used a planar architecture of $\mathrm{ITO} / \mathrm{TiO}_{2}-\mathrm{Cl} /$ perovskite/ $\mathrm{PDCBT} / \mathrm{MoO}_{\mathrm{x}} / \mathrm{Au}$ (Fig. 4a). Figure $4 \mathrm{~b}$ showed the statistical photovoltaic performance of the PSCs prepared in these three different conditions.

Compared with the tensile-strain and strain-free devices with average PCEs of 14.8 and $15.5 \%$, the compressive-strain devices exhibited higher average PCEs of $16.0 \%$. The best-performing devices of each type demonstrated AM1.5 PCEs of 15.14, 16.00, and $16.41 \%$ (Fig. $4 \mathrm{c}$ and Supplementary Fig. 16). Considering the wide bandgap of $\mathrm{CsPbI}_{2} \mathrm{Br}(1.92 \mathrm{eV})$, the performance of our compressive-strain devices is comparatively superior to that of strain-free $\mathrm{CH}_{3} \mathrm{NH}_{3} \mathrm{PbI}_{3}$-based devices with a bandgap of $1.64 \mathrm{eV}$ fabricated at room temperature ${ }^{14}$. Notably, all the devices exhibited high FFs above $80 \%$, especially the compressive-strain device with $85.1 \%$, comparable to those of the best-reported 
a

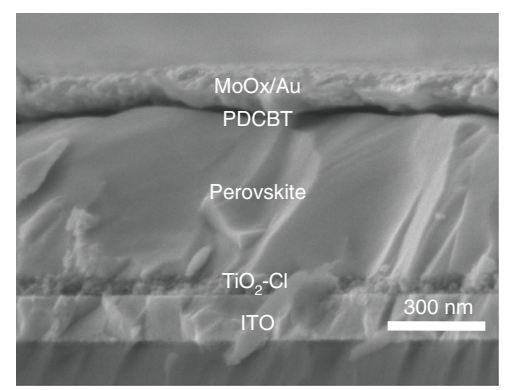

C

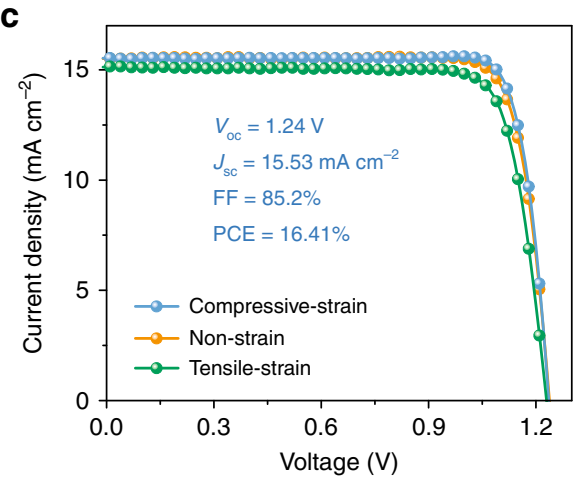

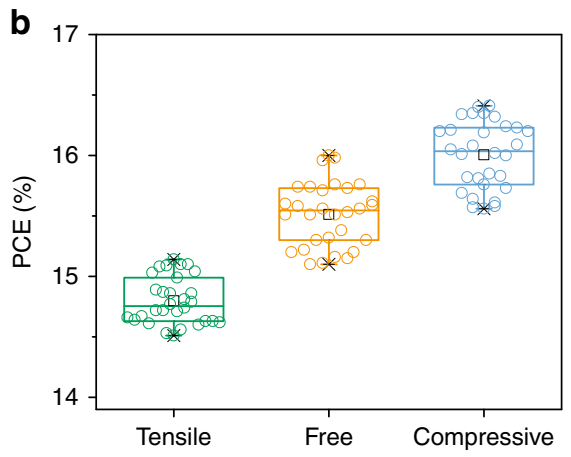

d

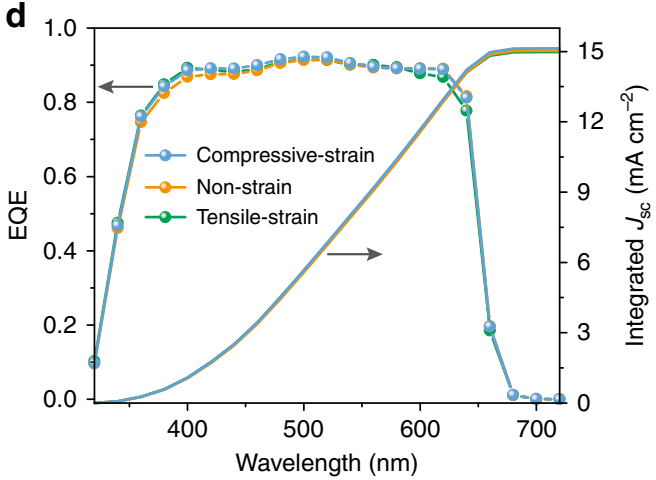

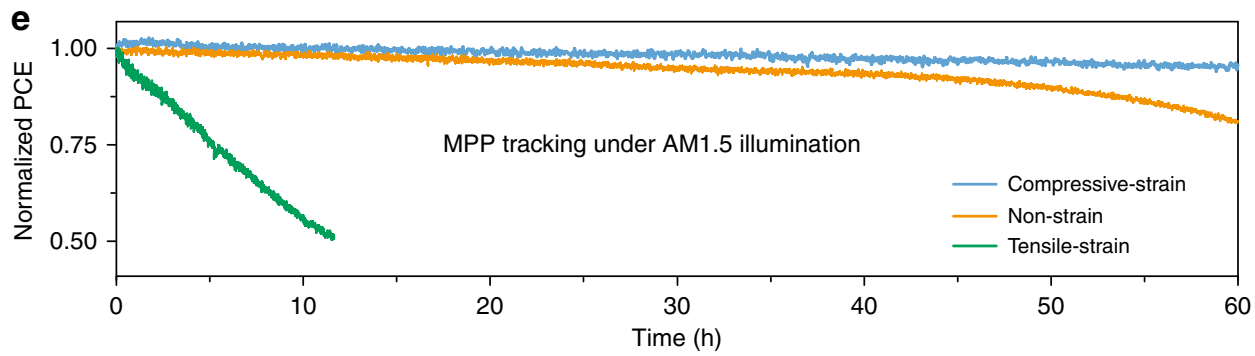

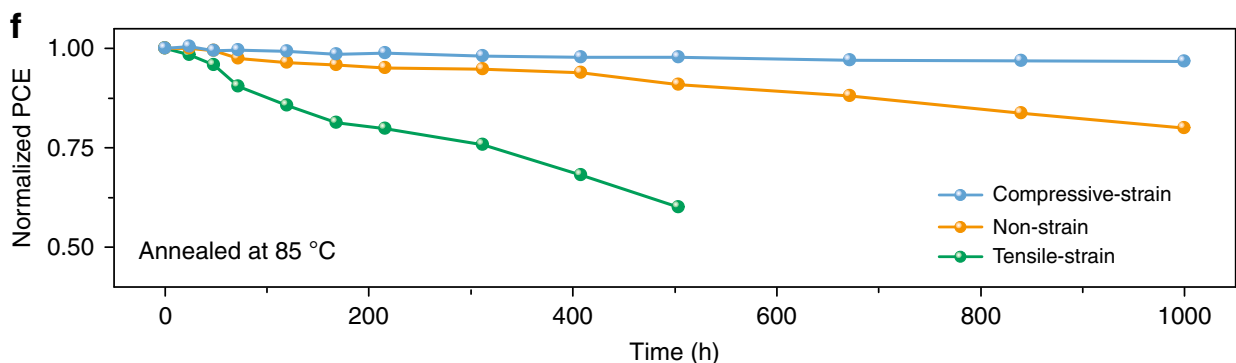

Fig. 4 Performance of PSCs under different strains. a Cross-sectional SEM image of PSCs. The scale bar is $300 \mathrm{~nm}$. $\mathbf{b}$ PCE statistics of 30 PSCs under each strain condition. The boxes indicate the 25th and 75th percentiles. The whiskers indicate the 5th and 95th percentiles. The cross symbols represent the maximum and minimum values. The median and mean are represented by the line dividing the boxes and the open square symbols, respectively. $\mathrm{c} J$ - $V$ curves of compressive-strain, strain-free and tensile-strain PSCs. d EQE curves of compressive-strain, strain-free and tensile-strain PSCs. e Evolution of normalized PCEs under MPP tracking and continuous simulated solar illumination $\left(100 \mathrm{~mW} \mathrm{~cm}^{-2}\right)$. $\mathbf{f}$ Evolution of normalized PCEs of PSCs kept at $85^{\circ} \mathrm{C}$ in a nitrogen atmosphere.

PSCs ${ }^{41-43}$, further confirming the efficient passivation and carrier extraction efficiency of PDCBT. The integrated photocurrent densities from EQE spectra (Fig. 4d) were consistent with the $J_{\mathrm{sc}}$ values measured from $J-V$ characterization (within $5 \%$ deviation).

Despite the comparable performances of PSCs under different strains, we found that the photostability under maximum power point (MPP) conditions and continuous one sun illumination varied greatly with strain (Fig. 4e). The compressive-strain and non-strain devices maintained 95 and $81 \%$ of their initial PCEs after continuous MPP operation for $60 \mathrm{~h}$, whereas the tensilestrain device showed a rapid loss of its 50\% initial PCE only after tens of hours, consistent with the observed photostability of perovskite films under different strains. We further tested the thermal stability of PSCs under continuous heating at $85^{\circ} \mathrm{C}$. The stability evolution was similar to the case under MPP condition (Fig. 4f). The tensile-strain device experienced significant PCE loss, while the compressive-strain and non-strain devices retained 96 and $80 \%$ of their initial PCEs after $1000 \mathrm{~h}$ of heating at $85^{\circ} \mathrm{C}$. The PCE loss of the tensile-strain device may be attributed to the phase segregation into Br-rich and I-rich perovskite phases, and subsequent transition of I-rich phase from photoactive to nonphotoactive phase ( $\alpha$ to $\delta)^{21}$. This result agrees with the observed 
thermal-stability of perovskite films under different strains ${ }^{11,12}$. Therefore, the extended photostability and thermal-stability of compressive-strain PSCs supports the contention that compressive strain can increase the activation energy for ion migration and suppress the halide ion migration in mixed-halide perovskites.

\section{Discussion}

In summary, we have introduced a strain-compensation strategy to reduce the thermally-induced tensile strain in perovskite films, by applying an external compressive strain produced by top functional layer. The high thermal expansion coefficient, strong binding affinity for the perovskite layer, and high processing temperature of the polymer HTL were found to be crucial parameters for this strain compensation strategy. Perovskite films with tunable strain (tensile to compressive) were successfully fabricated by adjusting the processing temperature of the HTL. We found that compressive strain can improve the intrinsic stability of perovskites, stemming from an increased activation energy for ion migration, and further enhance the photostability and thermal stability of state-of-art PSCs. This study provides an approach to regulate the strain in perovskite films, towards efficient and stable perovskite optoelectronic devices.

\section{Methods}

Solar cell fabrication. Pre-patterned indium oxide (ITO, TFD Devices)-coated glass slides were ultrasonically cleaned using acetone and isopropanol for $45 \mathrm{~min}$ each. The Cl-capped $\mathrm{TiO}_{2}\left(\mathrm{TiO}_{2}-\mathrm{Cl}\right)$ nanocrystal solution was synthesized according to previous work ${ }^{38}$. The $\mathrm{TiO}_{2}-\mathrm{Cl}$ layer was spin-coated twice on ITO substrate at $3000 \mathrm{rpm}$ for $30 \mathrm{~s}$ and then annealed on a hot plate at $150{ }^{\circ} \mathrm{C}$ for $30 \mathrm{~min}$ in ambient air. The substrates were immediately transferred to the $\mathrm{N}_{2}$-filled glovebox after cooling to room temperature. To make the $1.5 \mathrm{M} \mathrm{CsPbI}_{2} \mathrm{Br}$ precursor solution, $\mathrm{CsI}, \mathrm{PbI}_{2}$ and $\mathrm{PbBr}_{2}$ were mixed with a mole ratio of 2:1:1 in DMSO. The perovskite films were deposited onto the $\mathrm{TiO}_{2}-\mathrm{Cl}$ substrates with a two-step spin-coating procedure. The first step was $1000 \mathrm{rpm}$ for $10 \mathrm{~s}$ with an acceleration of $200 \mathrm{rpm} \mathrm{s}^{-1}$. The second step was $3000 \mathrm{rpm}$ for $90 \mathrm{~s}$ with a ramp-up of $1500 \mathrm{rpm} \mathrm{s}^{-1}$. The coated films were then annealed on hotplates in two steps: (i) $45^{\circ} \mathrm{C}$ for $3 \mathrm{~min}$; (ii) $160^{\circ} \mathrm{C}$ for $10 \mathrm{~min}$. PDCBT was dissolved in a mixture of chlorobenzene (CB) and 1,2-dichlorobenzene (ODCB) (1:1 by volume) at a concentration of $10 \mathrm{mg} / \mathrm{mL}$, and followed by stirring on a $60^{\circ} \mathrm{C}$ hot plate for $30 \mathrm{~min}$. For the PDCBT hot-casting process, the prepared perovskite film was first preheated to a desired temperature $\left(60-120^{\circ} \mathrm{C}\right)$ on a hot plate for $10 \mathrm{~min}$, and the PDCBT solution was also kept on a hot plate for $10 \mathrm{~min}$ at the same temperature as the perovskite film. The hot perovskite film was then immediately (within $5 \mathrm{~s}$ ) transferred to the spin coater chuck (which was at room temperature), and the hot PDCBT solution was dropped onto the hot film using a pipette almost simultaneously (Supplementary Fig. 17). The spin-coater was immediately started at a spin speed of $1000 \mathrm{rpm}$ for $20 \mathrm{~s}$. The thickness of PDCBT layer was about $50 \mathrm{~nm}$ (Supplementary Fig. 18). Finally, a $20 \mathrm{~nm}$ layer of $\mathrm{MoO}_{\mathrm{x}}$ and $100 \mathrm{~nm}$ layer of $\mathrm{Au}$ counter electrode were deposited on top of PDCBT by thermal evaporation in an Angstrom Engineering deposition system.

Materials and device characterization. XRD patterns were collected using a Rigaku D/Max-2500 diffractometer equipped with a $\mathrm{Cu} \mathrm{Kal}$ radiation $(\lambda=$ $1.54056 \AA$ ). XPS measurements were performed on an ESCALab220i-XL electron spectrometer (VG Scientific) using $300 \mathrm{~W}$ Al Ka radiation. Raman spectra (Horiba JobinYvon, LabRAM HR800) were measured under the excitation line of $532 \mathrm{~nm}$. Optical absorption measurements were carried out in a Lambda $950 \mathrm{UV} / \mathrm{Vis}$ spectrophotometer. PL spectra were measured using a Horiba Fluorolog time correlated single-photon-counting system with photomultiplier tube detectors. The excitation source was a laser diode at a wavelength of $374 \mathrm{~nm}$. The pulse duration is $110 \mathrm{ps}$, and peak power per area is $540 \mathrm{~W} \mathrm{~cm}^{-2}$. The light was illuminated from the glass side of the perovskite film. SEM images and EDS mapping were collected using Hitachi SU5000. For EDS mapping, the acceleration voltage was set at $5 \mathrm{kV}$ with spot intensity of 30 to increase the EDS signal to noise ratio. For secondary electron imaging, the acceleration voltage was kept at $3 \mathrm{kV}$ with spot intensity of 10 to minimize the beam damage. The $J-V$ characteristics of all the devices were measured using a Keithley 2400 source meter under AM1.5 G illumination (Newport, Class A). Unless otherwise stated, the $J$ - $V$ curves were measured in a $\mathrm{N}_{2}$ atmosphere with a scanning rate of $50 \mathrm{mV} \mathrm{s}^{-1}$ (voltage step of $10 \mathrm{mV}$ and delay time of $200 \mathrm{~ms})$. The active area was determined by the aperture shade mask $(0.049$ $\mathrm{cm}^{2}$ ) placed in front of the glass side of the solar cell to avoid overestimation of the photocurrent density. EQE measurements were performed using a Newport system (QuantX 300) with monochromatic light without any bias light. The system was calibrated by a certified Si solar cell (Newport) before the EQE measurement. The stability test was carried out at continuous MPP operation under AM1.5 G illumination by fixing the voltage at VMPP and then tracking the current output with a $420 \mathrm{~nm}$ cutoff UV filter. Thermal long-term stability tests were carried out by repeating the $J-V$ characterizations over various times. The devices were kept at $85^{\circ} \mathrm{C}$ in a nitrogen-filled glovebox.

Simulation methods. We used density-functional theory (DFT) performed in Vienna Ab Initio Simulation Package (VASP ${ }^{44}$ ) to study the perovskite crystal structure and migration of halide ions in $\mathrm{CsPbI}_{2} \mathrm{Br}$. Generalized gradient approximation (GGA) was considered with Perdew-Burke-Ernzerhof (PBE) generalized exchange-correlation functional ${ }^{45}$. The vdW correction of Grimmer's DFT $+\mathrm{D} 2^{46}$ was used to describe the weak interactions within the perovskite material while the plane-wave kinetic energy cutoff was fixed at $400 \mathrm{eV}$. The computational cell consisting of $64 \mathrm{CsPbI}_{2} \mathrm{Br}$ units ( 320 atoms) was employed in all calculations For structure relaxation, forces were converged to less than $0.01 \mathrm{eV} \AA^{-1}$. The Brillouin zone was sampled using a gamma-only wavevector grid and an electronic convergence criterion of $5 \times 10^{-7} \mathrm{eV}$ per formula unit was used. The halide vacancy defects were created by removing one halide atom from the 64 formulaunit computational cells. Activation energies for diffusion processes under differen biaxial strains were computed from the total energy difference between diffusing species in the ground-state configuration and at the saddle point of the diffusion process ${ }^{40}$. The migration mediated by halide ion vacancies was examined using nudged elastic band and constrained energy minimization methods. The migrating species was propagated along the migration direction in a series of small steps with all unconstrained degrees of freedom relaxed at each step.

Reporting summary. Further information on research design is available in the Nature Research Reporting Summary linked to this article.

\section{Data availability}

The data that support the findings of this study are available on reasonable request from the corresponding author.

Received: 10 December 2019; Accepted: 1 March 2020; Published online: 23 March 2020

\section{References}

1. Kojima, A., Teshima, K., Shirai, Y. \& Miyasaka, T. Organometal halide perovskites as visible-light sensitizers for photovoltaic cells. J. Am. Chem. Soc. 131, 6050-6051 (2009).

2. National Renewable Energy Laboratory. Best Research-cell Efficiencies https:// www.nrel.gov/pv/assets/pdfs/best-research-cell-efficiencies.20190923.pdf (2019).

3. Jiang, Q. et al. Surface passivation of perovskite film for efficient solar cells. Nat. Photon. 13, 460-466 (2019).

4. Grätzel, M. The light and shade of perovskite solar cells. Nat. Mater. 13, 838-842 (2014)

5. Wang, L. et al. A Eu${ }^{3+}-\mathrm{Eu}^{2+}$ ion redox shuttle imparts operational durability to Pb-I perovskite solar cells. Science 363, 265-270 (2019).

6. Wong-Stringer, M. et al. High-performance multilayer encapsulation for perovskite photovoltaics. Adv. Energy Mater. 8, 1801234 (2018).

7. Cheacharoen, R. et al. Design and understanding of encapsulated perovskite solar cells to withstand temperature cycling. Energy Environ. Sci. 11, 144-150 (2018).

8. Bella, F. et al. Improving efficiency and stability of perovskite solar cells with photocurable fluoropolymers. Science 354, 203-206 (2016).

9. Boyd, C. C., Cheacharoen, R., Leijtens, T. \& McGehee, M. D. Understanding degradation mechanisms and improving stability of perovskite photovoltaics. Chem. Rev. 119, 3418-3451 (2019).

10. Steele, J. A. et al. Thermal unequilibrium of strained black $\mathrm{CsPb}_{3}$ thin films Science 365, 679-684 (2019)

11. Zhao, J. et al. Strained hybrid perovskite thin films and their impact on the intrinsic stability of perovskite solar cells. Sci. Adv. 3, eaao5616 (2017).

12. Rolston, N. et al. Engineering stress in perovskite solar cells to improve stability. Adv. Energy Mater. 8, 1802139 (2018).

13. Zhu, C. et al. Strain engineering in perovskite solar cells and its impacts on carrier dynamics. Nat. Commun. 10, 815 (2019).

14. Zhang, H. et al. Toward all room-temprerature, solution-processed, highperformance planar perovskite solar cells: a new scheme of pyrideinepromoted perovskite formation. Adv. Mater. 29, 1604695 (2017)

15. Ge, C. et al. Ultralow thermal conductivity and ultrahigh thermal expansion of single-crystal organic-inorganic hybrid perovskite $\mathrm{CH}_{3} \mathrm{NH}_{3} \mathrm{PbX}_{3}(\mathrm{X}=\mathrm{Cl}, \mathrm{Br}$, I). J. Phys. Chem. C. 122, 15973-15978 (2018)

16. Keshavarz, M. et al. Tracking structural phase transitions in lead-halide perovskites by means of thermal expansion. Adv. Mater. 31, 1900521 (2019). 
17. Jacobsson, T. J., Schwan, L. J., Ottosson, M., Hagfeldt, A. \& Edvinsson, T. Determination of thermal expansion coefficients and locating the temperature-induced phase transition in methylammonium lead perovskites using X-ray diffraction. Inorg. Chem. 54, 10678-10685 (2015).

18. Burwig, T., Fränzel, W. \& Pistor, P. Crystal phases and thermal stability of coevaporated $\mathrm{CsPbX}_{3}(\mathrm{X}=\mathrm{I}, \mathrm{Br})$ thin films. J. Phys. Chem. Lett. 9, 4808-4813 (2018).

19. Wang, P. et al. Solvent-controlled growth of inorganic perovskite films in dry environment for efficient and stable solar cells. Nat. Commun. 9, 2225 (2018).

20. Wang, Y. et al. Thermodynamically stabilized $\beta-\mathrm{Cs} \mathrm{PbI}_{3}-$ based perovskite solar cells with efficiencies $>18 \%$. Science 365, 591-595 (2019).

21. Liu, M. et al. Lattice anchoring stabilizes solution-processed semiconductors. Nature 570, 96-101 (2019).

22. Liu, C. et al. Structurally reconstructed $\mathrm{CsPbI}_{2} \mathrm{Br}$ perovskite for highly stable and square-centimeter all-inorganic perovskite solar cells. Adv. Energy Mater. 9, 1803572 (2019).

23. Chen, W. et al. Precise control of crystal growth for highly efficient $\mathrm{CsPbI}_{2} \mathrm{Br}$ perovskite solar cells. Joule 3, 191-204 (2019).

24. Wang, K. et al. All-inorganic cesium lead iodide perovskite solar cells with stabilized efficiency beyond 15\%. Nat. Commun. 9, 4544 (2018).

25. Zeng, Z. et al. In situ grain boundary functionalization for stable and efficient inorganic $\mathrm{CsPbI}_{2} \mathrm{Br}$ perovskite solar cells. Adv. Energy Mater. 8, 1801050 (2018).

26. Tian, J. et al. Dual interfacial design for efficient $\mathrm{CsPbI}_{2} \mathrm{Br}$ perovskite solar cells with improved photostability. Adv. Mater. 31, 1901152 (2019).

27. Swarnkar, A. et al. Quantum dot-induced phase stabilization of $\alpha-\mathrm{CsPbI}_{3}$ perovskite for high-efficiency photovoltaics. Science 354, 92-95 (2016).

28. Xue, J. et al. Crystalline liquid-like behavior: surface-induced secondary grain growth of photovoltaic perovskite thin film. J. Am. Chem. Soc. 141, 13948-13953 (2019).

29. Hou, Y. et al. A generic interface to reduce the efficiency-stability-cost gap of perovskite solar cells. Science 358, 1192-1197 (2017).

30. Zhang, M., Guo, X., Ma, W., Ade, H. \& Hou, J. A polythiophene derivative with superior properties for practical application in polymer solar Cells. Adv. Mater. 26, 5880-5885 (2014).

31. Shen, X., Duzhko, V. V. \& Russell, T. P. A Study on the correlation between structure and hole transport in semi-crystalline regioregular P3HT. Adv. Energy Mater. 3, 263-270 (2013).

32. Wang, T., Pearson, A. J., Lidzey, D. G. \& Jones, R. A. L. Evolution of structure, optoelectronic properties, and device performance of polythiophene: fullerene solar cells during thermal annealing. Adv. Funct. Mater. 21, 1383-1390 (2011).

33. Joshi, S. et al. Bimodal temperature behavior of structure and mobility in high molecular weight P3HT thin films. Macromolecules 42, 4651-4660 (2009).

34. $\mathrm{Wu}, \mathrm{T}$. et al. Efficient defect passivation for perovskite solar cells by controlling the electron density distribution of donor- $\pi$-acceptor molecules. Adv. Energy Mater. 9, 1803766 (2019).

35. Lin, $Y$. et al. $\pi$-conjugated Lewis base: efficient trap-passivation and chargeextraction for hybrid perovskite solar cells. Adv. Mater. 29, 1604545 (2017).

36. Zheng, X. et al. Defect passivation in hybrid perovskite solar cells using quaternary ammonium halide anions and cations. Nat. Energy 2, 17102 (2017).

37. Saidaminov, M. I. et al. Suppression of atomic vacancies via incorporation of isovalent small ions to increase the stability of halide perovskite solar cells in ambient air. Nat. Energy 3, 648-654 (2018).

38. Tan, H. et al. Efficient and stable solution-processed planar perovskite solar cells via contact passivation. Science 355, 722-726 (2017).

39. Ferdani, D. W. et al. Partial cation substitution reduces iodide ion transport in lead iodide perovskite solar cells. Energy Environ. Sci. 12, 2264-2272 (2019).

40. Eames, C. et al. Ionic transport in hybrid lead iodide perovskite solar cells. Nat. Commun. 6, 7497 (2015).

41. Chiang, C.-H. \& Wu, C.-G. A method for the preparation of highly oriented $\mathrm{MAPbI}_{3}$ crystallites for high-efficiency perovskite solar cells to achieve an $86 \%$ fill factor. ACS Nano 12, 10355-10364 (2018).

42. Wu, C.-G. et al. High efficiency stable inverted perovskite solar cells without current hysteresis. Energy Environ. Sci. 8, 2725-2733 (2015).
43. Yang, Y. et al. Top and bottom surfaces limit carrier lifetime in lead iodide perovskite films. Nat. Energy 2, 16207 (2017).

44. Kresse, G. \& Furthmüller, J. Efficient iterative schemes for ab initio totalenergy calculations using a plane-wave basis set. Phys. Rev. B 54, 11169-11186 (1996).

45. Perdew, J. P., Burke, K. \& Ernzerhof, M. Generalized gradient approximation made simple. Phys. Rev. Lett. 77, 3865-3868 (1996).

46. Press, W. H. Numerical Recipes: The Art of Scientific Computing (Cambridge University Press, Cambridge, 2007).

\section{Acknowledgements}

This publication is based in part on work supported by the US Department of the Navy, Office of Naval Research (Grant Award No. N00014-17-1-2524), the Ontario Research Fund Research Excellence Program, and by the Natural Sciences and Engineering Research Council (NSERC) of Canada. D.-J.X. acknowledges the support of the National Natural Science Foundation of China (21922512, 21875264), and the Youth Innovation Promotion Association CAS (2017050).

\section{Author contributions}

D.-J.X. and Y.H. conceived the idea, prepared films, fabricated all devices and characterized them. S.-C.L. assisted in X.R.D. and X.P.S. measurements. M.W., B.C., and Z.H assisted in PL and SEM measurements. Z.L. performed the DFT calculations and analyzed the results. B.S., Y.D., and M.I.S. assisted with the discussions. S.O.K. and J.-S.H. assisted with the manuscript preparation. E.H.S. directed the overall research. D.-J.X., Y.H., A.H.P., and E.H.S. wrote the manuscript. All authors read and commented on the manuscript.

\section{Competing interests}

The authors declare no competing interests.

\section{Additional information}

Supplementary information is available for this paper at https://doi.org/10.1038/s41467020-15338-1.

Correspondence and requests for materials should be addressed to E.H.S.

Peer review information Nature Communications thanks Masoumeh Keshavarz and the other, anonymous, reviewer(s) for their contribution to the peer review of this work. Peer reviewer reports are available.

Reprints and permission information is available at http://www.nature.com/reprints

Publisher's note Springer Nature remains neutral with regard to jurisdictional claims in published maps and institutional affiliations.

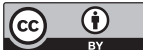

Open Access This article is licensed under a Creative Commons Attribution 4.0 International License, which permits use, sharing, adaptation, distribution and reproduction in any medium or format, as long as you give appropriate credit to the original author(s) and the source, provide a link to the Creative Commons license, and indicate if changes were made. The images or other third party material in this article are included in the article's Creative Commons license, unless indicated otherwise in a credit line to the material. If material is not included in the article's Creative Commons license and your intended use is not permitted by statutory regulation or exceeds the permitted use, you will need to obtain permission directly from the copyright holder. To view a copy of this license, visit http://creativecommons.org/ licenses/by/4.0/.

(c) Crown 2020 